e-J. Surf. Sci. Nanotech. Vol. 3 (2005) 427-432

Conference - ISSS-4 -

\title{
Structure and Bonding of Trace Ni Catalyst in Carbon Nanotube Studied by Ni K-Edge XANES *
}

\author{
Mayuko Ushiro ${ }^{\dagger}$ \\ Graduate School for Science, Chiba University, Yayoi-cho 1-33, Inage-ku, Chiba 263-8522, Japan, \\ Kiyotaka Asakura \\ Catalyst Research Center, Hokkaido University, Kita-ku, Sapporo, Hokkaido 001-0021, Japan,
}

Kenryo Ohminami, Shin-ichi Nagamatsu, and Takashi Fujikawa

Graduate School for Science, Chiba University, Yayoi-cho 1-33, Inage-ku, Chiba 263-8522, Japan

(Received 14 October 2005; Accepted 28 November 2005; Published 9 December 2005)

\begin{abstract}
We carry out Ni K-edge X-ray Absorption Near Edge Structure (XANES) analyses to study local electronic and geometric structure around $\mathrm{Ni}$ impurities after $\mathrm{HCl}$ treatment in carbon nanotubes (CNTs) by applying full multiple scattering calculations. For the trace Ni species in CNT after the treatment, we consider possible models consistent with Ni-C distance and coordination number estimated by previous Extended X-ray Absorption Fine Structure (EXAFS) analyses. The present analyses allow us to distinguish between two defect models for the Ni location; a crack-like defect and Stone-Wales defect. We also find that the curvature of CNTs affects the calculated XANES spectra, which can provide useful information about outer or inner adsorption on CNT walls. Ab initio density functional calculation supports the presence of Ni atoms at the outside of the nanotube.

[DOI: $10.1380 /$ ejssnt.2005.427]
\end{abstract}

Keywords: Carbon nanotube; Nickle; XANES; Multiple scattering calculation;

\section{INTRODUCTION}

Carbon nanotubes (CNTs) and carbon nanofibers (CNFs) can be applied to electric devices, hydrogen reservoir, medical usages and so on. There are some preparation methods to produce CNTs/CNFs, and metal catalysts such as $\mathrm{Ni}$ are widely used. Although most of $\mathrm{Ni}$ in the CNTs/CNFs can be removed by an acid (e.g. $\mathrm{HCl}$ ) treatment, a small amount of $\mathrm{Ni}$ impurities are left in the CNTs/CNFs. Therefore, small amount of toxic Ni may be a serious problem to use CNTs/CNFs as living materials. Information on the structure and the chemical state of trace $\mathrm{Ni}$ species are important to the medical application of CNTs/CNFs. However, we have had no definite conclusion for the location of the metal atoms due to the lack of direct experimental evidence about the local structure around the Ni impurities.

Recently, Asakura et al. reported the first Extended X-ray Absorption Fine Structure (EXAFS) and X-ray Absorption Near Edge Structure (XANES) spectra of Ni species in CNFs before and after $\mathrm{HCl}$ treatment.[1] Before the $\mathrm{HCl}$ treatment, the XANES spectrum is quite similar to that of $\mathrm{Ni}$ foil, whereas the spectrum after the treatment has two specific peaks in the absorption edge region and suggests that the Ni impurities (a few hundred ppm) should not be metal $\mathrm{Ni}$ particles or simple Ni oxides.

On the other hand, there are some theoretical work undertaken for the study of interaction of $3 \mathrm{~d}$ transition metal atoms and dimers with a single-walled ideal (no defect) CNTs. A density functional calculation shows that outside adsorption sites are most favorable for Ni-doped

\footnotetext{
* This paper was presented at International Symposium on Surface Science and Nanotechnology (ISSS-4), Saitama, Japan, 14-17 November, 2005.

${ }^{\dagger}$ Corresponding author: m.ushiro@graduate.chiba-u.jp
}

$(4,4)$ nanotubes at atop sites with Ni-C distance $1.87 \AA$.[2] A different approach using pseudo potential plane wave method has predicted rather large binding energy for $\mathrm{Ni}$ coating on the CNTs.[3] These two studies are on the $\mathrm{Ni}$ adsorption on the ideal (no defect) CNTs. Andriotis et al. investigate catalytic action of $\mathrm{Ni}$ atoms in the growth of single-walled CNTs using tight-binding molecular dynamics in conjunction with ab initio total energy calculations. [4] Their simulations favor the Ni atom acting initially as defect stabilizers and subsequently diffusing to a defect positions on exterior ring.

XANES spectra provide longer range information (typically $\approx 5 \AA$ ) than EXAFS spectra, and stereochemical information. [5, 6] EXAFS analyses are rather easy compared with XANES analyses. The XANES, however, has an important advantage over the EXAFS: Even weak electron scatterers carbon still provides useful information in the XANES analyses.[6]

Main purpose of this paper is to obtain some useful information on the local structure and bonding of trace Ni species in carbon nanotube by full multiple scattering XANES analyses [7-11] and ab initio molecular orbital theory. The molecular orbital theory gives us some useful information about the electronic structure and the optimized structure of a $\mathrm{Ni}$ atom trapped at a crack-like defect site of the graphene sheet.

\section{THEORY}

The XANES theory used in this paper is based on the short-range-order full multiple scattering theory proposed by Fujikawa et al. [7] Later, this theory was modified by a partitioning technique in order to reduce the computation time.[8-11] Here, we summarize the theoretical methods.

The X-ray absorption intensity $\sigma$ from the core orbital $\phi_{c}(\mathbf{r})=R_{l_{c}}(r) Y_{L_{c}}(\hat{\mathbf{r}}), L_{c}=\left(l_{c}, m_{c}\right)$ at site A (X-ray absorbing atom) is given by Eq.(1) for photoelectron kinetic 
energy $\epsilon_{k}=k^{2} / 2$. We assume excitation by a linearly polarized X-ray in the z-direction, [8]

$$
\begin{aligned}
\sigma= & -\frac{8}{3} \operatorname{Im}\left\{\sum_{L, L^{\prime}, m_{c}} i^{l-l^{\prime}} \exp \left\{i\left(\delta_{l}^{A}+\delta_{l^{\prime}}^{A}\right)\right\} \rho_{c}(l) \rho_{c}\left(l^{\prime}\right)\right. \\
& \left.\times G\left(L_{c} 10 \mid L\right) G\left(L_{c} 10 \mid L^{\prime}\right)\left(t^{-1}\right)_{L L}^{A A}\left[(1-X)^{-1}\right]_{L L^{\prime}}^{A A}\right\},
\end{aligned}
$$

where $G\left(L L^{\prime} \mid L^{\prime \prime}\right)$ is Gaunt's integral and $\rho_{c}(l)$ is the radial dipole integral between the radial part $R_{l_{c}}(r)$ of $\phi_{c}(\mathbf{r})$ and the $l$-th partial wave of photoelectrons $R_{l}(r)$ at site A. The phase shift of the $l$-th partial wave at site $A$ is represented by $\delta_{l}^{A}$. We introduce the matrix $X$ specified with site index $\alpha$ and angular momentum $L$ and so on; it is defined as

$$
X_{L L^{\prime}}^{\alpha \beta}=t_{l}^{\alpha} G_{L L^{\prime}}\left(\mathbf{R}_{\alpha}-\mathbf{R}_{\beta}\right)\left(1-\delta_{\alpha \beta}\right),
$$

where $t_{l}^{\alpha}$ and $G_{L L^{\prime}}$ represent the $T$-matrix at site $\alpha$ and the Green's function in an angular momentum representation. The inverse matrix $(1-X)^{-1}$ includes an infinite order of the full multiple scattering inside the cluster we are considering. The phase shift in $t_{l}^{\alpha}\left(=-\left[\exp \left(2 i \delta_{l}^{\alpha}\right)-1\right] / 2 i k\right)$ is one of the most important features and reflects the electronic structure of the surrounding atoms, which is calculated within the Hartree-Fock approximation. The Green's function $G_{L L^{\prime}}$ reflects the geometrical structure. The clusters used in the present work include all surrounding atoms up to about $7 \AA$ except the carbide model (up to about $5 \AA$ ) around an X-ray absorption atom.

\section{RESULTS AND DISCUSSION}

\section{A. Multiple Scattering Analyses of Ni K-Edge XANES}

At first we study the Ni adsorption structure on CNTs by use of the multiple scattering analyses. So far no experimental XANES for Ni impurities in CNTs has been reported, but those for $\mathrm{Ni}$ impurities in CNFs have been reported.[1, 12] As shown later, the calculated XANES spectra for CNFs and CNTs are very similar, so that we can safely refer to the XANES for the Ni impurities in CNFs to investigate the local structure around $\mathrm{Ni}$ impurities in CNTs.

Figure 1 shows the K-edge XANES spectra of Ni species in the CNFs before and after the $\mathrm{HCl}$ treatment together with that of $\mathrm{Ni}$ foil.[1] The XANES spectrum after the treatment shows a marked difference from that for before the treatment which is quite similar to that for $\mathrm{Ni}$ foil. Having two specific peaks in the absorption edge region, the spectrum after the treatment suggests that the trace $\mathrm{Ni}$ species should be different from metal Ni particles or simple Ni oxides.[1] In addition, this characteristic peaks should be the fingerprint for the calculated XANES spectra using multiple scattering theory. We investigate the following possible models for the structure of $\mathrm{Ni}$ in $\mathrm{CNF}$ and CNT.

$$
\text { - an edge model }
$$

\section{Ni K-edge XANES spectra}

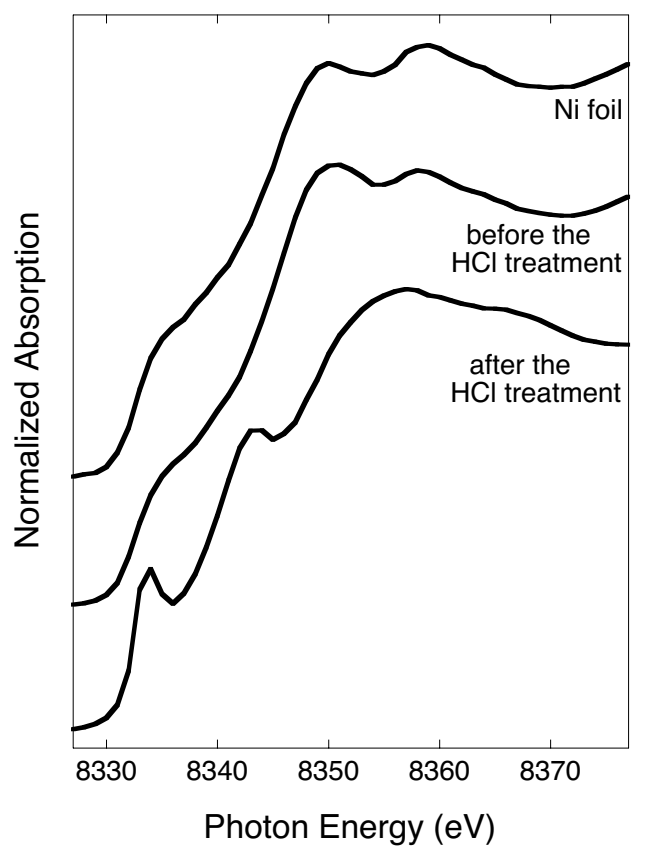

FIG. 1: The experimental Ni K-edge XANES spectra of Ni species in the carbon nanofibers before and after the $\mathrm{HCl}$ treatment together with that of $\mathrm{Ni}$ foil.[1]

- a substitution model

- a Stone-Wales model

In these calculations, we use a flat graphene sheet for simplicity.

\section{1. an edge model}

Sharp has applied organometallic chemistry at the edge of polycyclic aromatic hydrocarbons to the step-growth synthesis of single-walled CNTs.[13] Ni atoms can form covalent bonds with carbon atoms at edges of graphene sheets because of having dangling bonds. We study the edge model shown in Fig.2 (a) where Ni-C distance is 1.8 $\AA$ with coordination number 1 . The bond distance is consistent with the EXAFS result $1.83 \pm 0.05 \AA$, though the coordination number is much smaller than the observed one, $2.4 \pm 0.8$.[1] The calculated Ni K-edge XANES spectrum is shown in Fig.3 (a) compared with the experimental spectrum (broken line). The calculated result shows too small shoulders at 7 and $16 \mathrm{eV}$ compared with the specific peaks after the treatment, and too rapid decrease above $30 \mathrm{eV}$ : This model fails to explain the observed features. We thus have no need to consider this model anymore.

\section{2. defect models}

$\mathrm{Ni}$ can be in a defect site in a graphene sheet. Meng et al. applied Hartree-Fock calculations by use of approximate exchange potential.[14] Their results show strong attractive interaction and bonding with CNTs due to the 
(a)

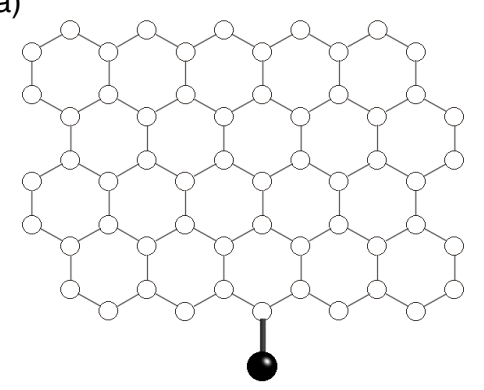

(c)

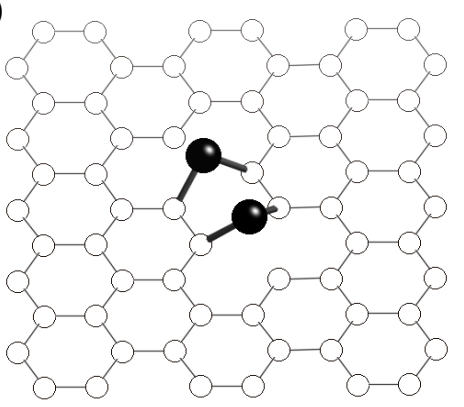

(b)

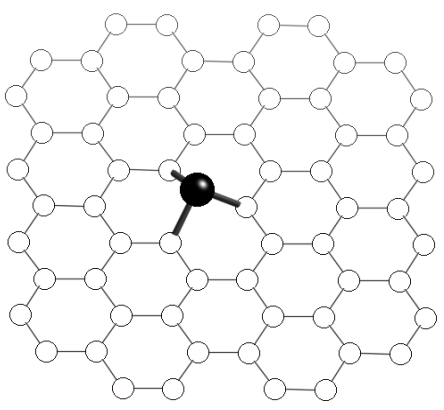

FIG. 2: An edge model (a) and two crack-like defect models (substitution models) (b) and (c) where black (white) balls are Ni (carbon) atoms. In (a) a Ni atom forms covalent bond with a nearest neighbor edge carbon with Ni-C distance $1.8 \AA$ An In (b) one $\mathrm{Ni}$ atom binds to three nearby carbon atoms in graphene sheet where $\mathrm{Ni}-\mathrm{C}$ distance is $1.8 \AA$, and in (c) two Ni atoms bind two nearby carbon atoms in graphene sheet with Ni-C (Ni-Ni) distance $1.8 \AA(2.5 \AA)$.

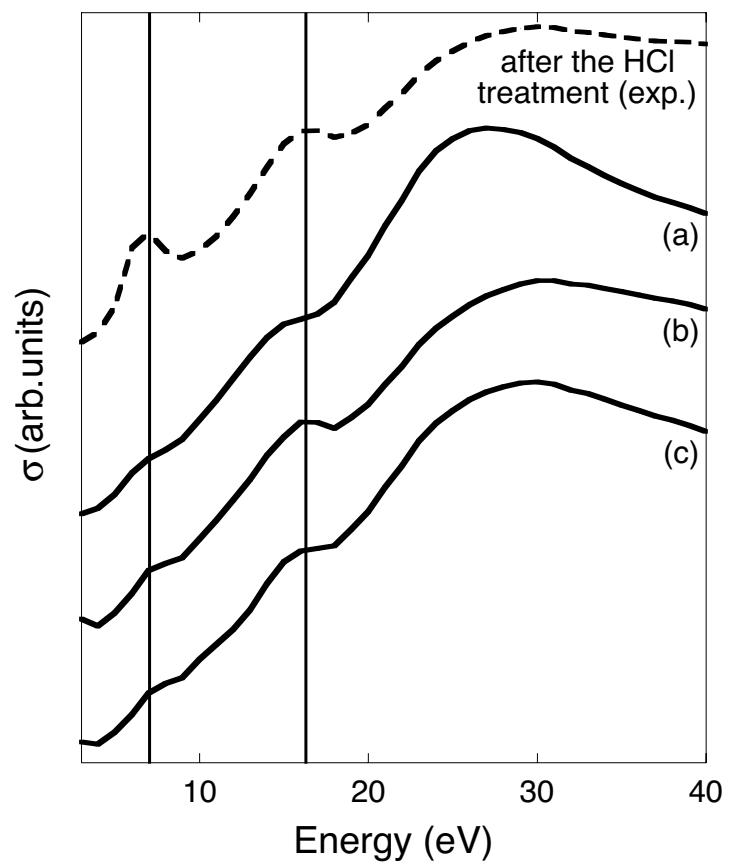

FIG. 3: Comparison of the calculated Ni K-edge XANES spectra for the three models shown in Fig.2 (a) an edge model and (b),(c) substitution models and the experimental spectrum after the treatment (broken line). The energy is measured from the onset of the K-edge absorption.

unfilled 3d shell for transition metals. A different theoretical work also supports the $\mathrm{Ni}$ adsorption at defect sites. [4] We thus investigate two types of defects, crack-like defects and Stone-Wales defects.

First we consider two crack-like defect models, substitution models, shown in Figs.2 (b) and (c). In the model (b), one Ni atom forms Ni-C covalent bonds with the Ni-C distance $1.8 \AA$ and coordination number 3 . In the model (c), two Ni atoms bind to $\mathrm{C}$ atoms at crack-like defect sites of a graphene sheet with the same distance with coordination number 2 , and $\mathrm{Ni}-\mathrm{Ni}$ distance is $2.5 \AA$ with coordination number 1 . Our recent EXAFS analyses show that the coordination number of $\mathrm{C}$ around $\mathrm{Ni}$ is 2.5.[12] Therefore the first model is not in contradiction to this results, but the latter can be ruled out as shown below. Figures 3 (b) and (c) show the calculated XANES spectra for the substitution models shown in Figs.2 (b) and (c) compared with the experimental spectrum after the treatment (broken line). Although the model (b) well explains the two characteristic peaks in the experimental data, the dimer model (c) gives too small peak at $\sim 7 \mathrm{eV}$ and rapid decrease above $30 \mathrm{eV}$ in comparison with the monomer model (b). The peak at $7 \mathrm{eV}$ is located just at the beginning of the edge rise and should have a contribution from atomic bound state that can not be fully taken into account by the present method. We thus expect that the model (b) can be a good candidate for trace Ni species.

Some CNTs are composed of multilayer with interlayer distance $3.4 \AA$ A. $[15,16]$ We add another graphene sheet under the model (b) shown in Fig.2. This model gives quite similar XANES spectrum to Fig.3 (b): The next-layer gives rise to negligible effect on the calculated spectrum.

Another type of defect, the Stone-Wales defect, has a pair of 5-7 rings which can be created by rotating a C-C bond in the hexagonal network by $90^{\circ}$.[17] Recent molecular orbital calculations show that the introduction of Stone-Wales defect would only benefit the adsorption capacity of B, N, F and Si among 10 foreign atoms $(H$, B, C, N, O, F, Si, P, Li and Na).[18] This result suggests that the Stone-Wales defects could be studied for the Ni adsorption site. We calculate Ni K-edge XANES spectra for four Stone-Wales models where one or two Ni atoms are adsorbed on the 5 or 7 rings of the Stone-Wales defect site. These models, however, fail to explain the two peaks 


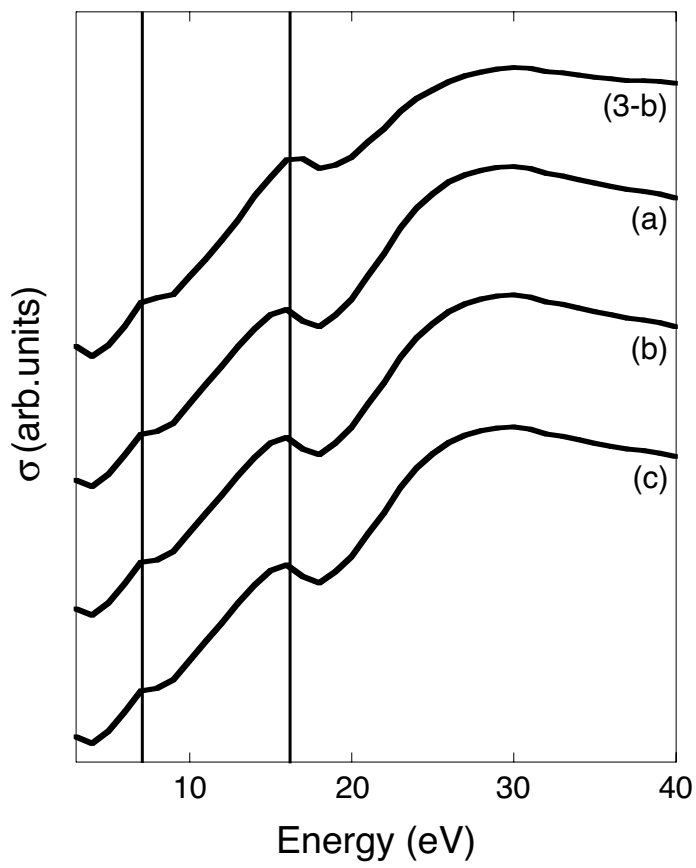

FIG. 4: The calculated XANES spectra for three different CNT models, (a) armchair, (b) zig-zag, and (c) chiral models compared with that (3-b) for the flat model shown by Fig.2 (b).

at 7 and $16 \mathrm{eV}$ because the calculated spectra of these models show structureless spectrum up to about $25 \mathrm{eV}$. We thus can rule out the Stone-Wales models.[12]

\section{3. influence of detailed CNT structures}

CNTs can be classified by the rolling up way of the graphene sheet. In the graphene honeycomb lattice, the unit cell is spanned by the two vector $\mathbf{a}_{1}$ and $\mathbf{a}_{2}$, and contains two carbon atoms at the position $\frac{1}{3}\left(\mathbf{a}_{1}+\mathbf{a}_{2}\right)$ and $\frac{2}{3}\left(\mathbf{a}_{1}+\mathbf{a}_{2}\right)$, where the basis vectors of length $\left|\mathbf{a}_{1}\right|=\left|\mathbf{a}_{2}\right|=$ $2.46 \AA$ form an angle of $60^{\circ}$. A graphene lattice vector $\mathbf{c}=n_{1} \mathbf{a}_{1}+n_{2} \mathbf{a}_{2}$ becomes the circumference of the tube, and it is usually denoted by the pair of integers called chiral vector $\left(n_{1}, n_{2}\right)$. In particular $(n, n)$ tubes are called armchair tubes and $(n, 0)$ tubes are called zig-zag tubes. Tubes of type $(n, m)$ are called chiral tubes.[15] We thus investigate the substitution model adsorbed on outside of the CNT tube (see Fig.5 (b)). Figure 4 shows the calculated XANES spectra for three different models depending on three different structures of CNTs with $14 \AA$ in diameter: (a) armchair, (b) zig-zag, and (c) chiral models. They are compared with the spectrum Fig.3 (b) for the flat model shown in Fig.2 (b). Comparing the three spectra, we understand the differences of the tube structure have very small influence on the calculated XANES spectra. The calculated XANES spectrum for the flat model only shows small difference from those for these tube models. This may be because of the similar local structure around the Ni atom. As the overall XANES is not so sensitive to the curvature, the XANES for CNF and CNT can show similar spectra. Detailed analyses, however, can provide information on outer or inner adsorption as discussed below. (a)

(b)
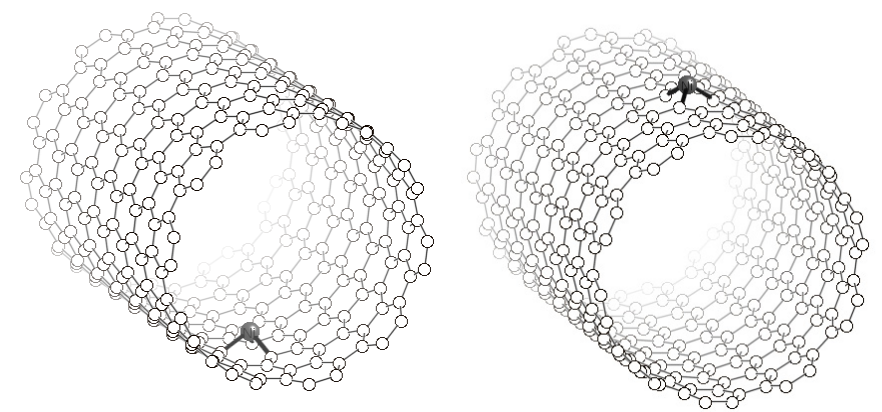

(c)

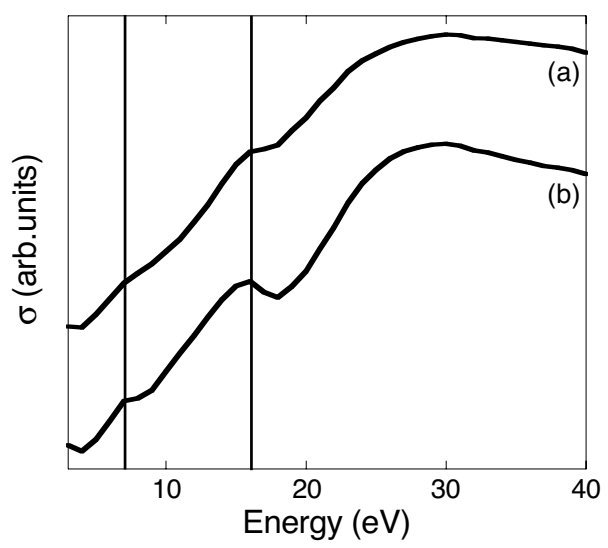

FIG. 5: Two tube models. The tube is armchair $(10,10)$ with $14 \AA$ in diameter. In model (a) a Ni atom occupies the inner substitution site, whereas it occupies the outer site in model (b), where a black (white) ball is Ni (carbon) atom. The calculated XANES spectra for these models are shown in (c).

We also check how the curvature of CNTs has some influence on the XANES spectra by using the multiple scattering theory. We thus postulate CNT models with $14 \AA$ in diameter shown in Figs.5 (a) and (b). In the model (a), one $\mathrm{Ni}$ atom is inside the tube, and in the model (b) outside. The calculated Ni K-edge XANES spectra are shown in Fig.5 (c). The "inside model" gives weaker peaks at 7 and $16 \mathrm{eV}$ than the "outside model". This may be because the different distances to carbon atoms in the next neighbors in these models affect the XANES spectra. This result is quite interesting because any other experimental tools cannot provide such detailed information.

\section{B. Molecular Orbital Analyses}

In order to obtain useful information about the electronic structures, we apply the $a b$ initio molecular orbital theory to these $\mathrm{Ni}$ adsorption models.

First, we optimize the structure of the substitution model by using Gaussian 03 code [19] for a $\mathrm{NiC}_{41} \mathrm{H}_{16}$ cluster. In order to calculate the optimized structure, we use a density functional theory (DFT) method employed the B3LYP type exchange-correlation potential. The cal- 


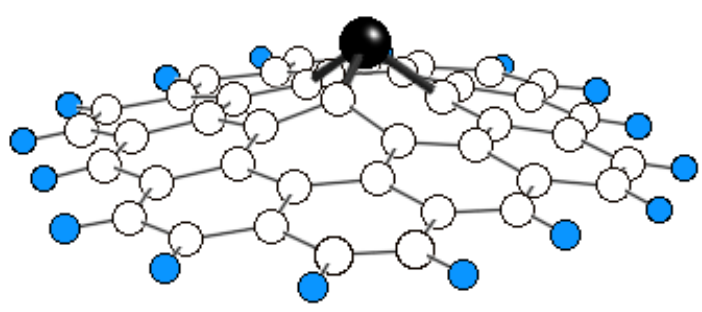

FIG. 6: The optimized structure of substitution model, where a Ni atom is in a crack-like defect site of a graphene sheet, calculated by DFT method.[19] The Ni atom occupies the outside of CNT with Ni-C distance $1.85 \AA$. In this figure a black ball is $\mathrm{Ni}$ atom and white (blue) balls are carbon (hydrogen) atoms.

TABLE I: Population analyses of substitution model and $\mathrm{Ni}(\mathrm{CO})_{4}$ by DFT calculation.

\begin{tabular}{|c|c|c|c|c|c|}
\hline \multicolumn{3}{|c|}{ substitution model } & \multicolumn{3}{|c|}{$\mathrm{Ni}(\mathrm{CO})_{4}$} \\
\hline \multirow[t]{4}{*}{$\mathrm{Ni}$} & total & +0.08 & $\mathrm{Ni}$ & total & +0.38 \\
\hline & 3d & 8.93 & & $3 \mathrm{~d}$ & 9.17 \\
\hline & $4 \mathrm{~s}$ & 0.73 & & $4 \mathrm{~s}$ & 0.45 \\
\hline & $4 \mathrm{p}$ & 0.27 & & $4 \mathrm{p}$ & 0.02 \\
\hline \multirow[t]{3}{*}{$\mathrm{C}^{a}$} & total & -0.05 & C & total & +0.34 \\
\hline & $2 \mathrm{~s}$ & 1.23 & & $2 \mathrm{~s}$ & 1.52 \\
\hline & $2 \mathrm{p}$ & 2.82 & & $2 \mathrm{p}$ & 2.13 \\
\hline \multirow[t]{3}{*}{$\mathrm{C}^{b}$} & total & $\sim 0.0$ & $\mathrm{O}$ & total & -0.44 \\
\hline & $2 \mathrm{~s}$ & 1.1 & & $2 \mathrm{~s}$ & 1.73 \\
\hline & $2 \mathrm{p}$ & 2.9 & & $2 \mathrm{p}$ & 4.70 \\
\hline
\end{tabular}

${ }^{a}$ nearest neighbor carbon atoms

${ }^{b}$ other carbon atoms

culation is performed by LanL2MB basis set. The optimized structure of $\mathrm{Ni}$ adsorption is obtained by use of the initial flat (CNF) structure as shown by Fig.2 (b). The optimized structure is shown in Fig.6, where the Ni occupies the outside of the curved CNT sheet. This result supports "outside model" shown in Fig.5 (b). The Ni-C bonds are $1.85 \AA$, which is close to Ni-C covalent bond length $(1.85 \AA)$ of $\mathrm{Ni}(\mathrm{CO})_{4}$ obtained by the same calculation method. This result is also close to the observed Ni-C bond length of $\mathrm{Ni}(\mathrm{CO})_{4}(1.82 \AA)$.[20] Therefore, Ni impurities is strongly bound to the sheet by the covalent bond. This result is consistent with the result of EXAFS analyses.[1]

Second, we study the bonding character of the $\mathrm{Ni}-\mathrm{C}$ bonds by using natural population analysis.[21] The natural atomic-orbital occupancies are tabulated in TABLE 1 . The Ni and the carbons are almost neutral in the substitution model, while the $\mathrm{Ni}$ and $\mathrm{C}$ atoms are positively charged, and $\mathrm{O}$ atoms are negatively charged in $\mathrm{Ni}(\mathrm{CO})_{4}$. The bond order between the $\mathrm{C}$ atoms bound to the $\mathrm{Ni}$ atom and the next neighbor $\mathrm{C}$ atoms is 1.3 , a little smaller than 1.5 in graphene sheets, which is related to the distortion from the flat carbon arrangement in graphene sheets. More detailed analyses will be give in the forthcoming paper.

\section{CONCLUSION}

In this paper, we calculated XANES spectra to determine the local structure of trace Ni species in CNT. The substitution model well explains the observed two specific peaks in the absorption edge region after the $\mathrm{HCl}$ treatment. Any other models fail to predict the observed features in the XANES spectrum after the treatment. More detailed analyses show the curvature of a graphene sheet affects the XANES spectra, and the XANES analyses can provide useful information whether $\mathrm{Ni}$ adsorbs inside or outside of a CNT tube.

We also study geometric and electronic structures of the substitution model by using ab initio molecular orbital theory. The optimized structure favor the "outside model" of Ni adsorption on CNTs. The present MO calculation shows that the $\mathrm{Ni}$ and $\mathrm{C}$ atoms are almost neutral in the substitution model.

This work demonstrates the remarkable usefulness of the XAFS (XANES+EXAFS) study combined with multiple scattering calculations for very dilute systems (about a few hundred ppm), Ni in graphene sheets. Other approaches would presumably be difficult to provide such detailed structural information around dilute Ni impurities.

\section{Acknowledgments}

The authors are grateful to Prof. K. Kaneko, Prof. H. Kano, Dr. Y. Hattori, K. Uno and T. Akatsuka for their valuable comments on this work.
[1] K. Asakura, W. J. Chun, K. Tohji, Y. Sato and F. Watari, Chem. Lett. 34, 382 (2005).

[2] Y. Yagi, T. M. Brier, M. H. F. Sluiter, V. Kummar, A. A. Farajian and Y. Kawazoe, Phys. Rev. B 69, 075414 (2004).

[3] E. Durgun, S. Dag, V. M. K. Bagci, O. Gülseren, T. Yildirim and S. Ciraci, Phys. Rev. B 67, 201401(R) (2003).

[4] A. N. Andriostis, M. Menon and F. Froudakis, Phys. Rev. Lett. 85, 3193 (2000).

[5] J. J. Rehr and R. C. Albers, Rev. Mod. Phys. 72, 621 (2000).

[6] K. Uno, Y. Notoya, T. Fujikawa, H. Yoshikawa and K. Nishikawa, Jpn J. Appl. Phys. 44, 4073 (2005).
[7] T. Fujikawa, T. Matsuura and H. Kuroda, J. Phys. Soc. Jpn. 52, 905 (1983).

[8] T. Fujikawa, J. Phys. Soc. Jpn. 62, 2155 (1993).

[9] T. Fujikawa and N. Yiwata, Surf. Sci. 357, 60 (1996).

[10] T. Fujikawa, R. Yanagisawa, N. Yiwata and K. Ohtani, J. Phys. Soc. Jpn. 66, 257 (1997).

[11] T. Fujikawa, K. Nakamura, S. Nagamastu and J. J. Rehr, J. Phys. Soc. Jpn. 71, 357 (2002).

[12] M. Ushiro, K. Uno, Y. Sato, K. Tohji, F. Watari, W. J. Chun, Y. Koike, K. Asakura and T. Fujikawa, unpublished.

[13] P. R. Sharp, J. Organometallic Chem. 683, 288 (2003).

[14] F. Y. Meng, L. G. Zhou, S. Q. Shi and R. Yang, Carbon 41, 2023 (2002). 
[15] S. Reich, C. Thomsen and J. Maultzsch, Carbon Nanotube. Basic Concepts and Physical Properties, (2004).

[16] Y. Sato, K. Shibata, H. Kataoka, S. Ogino, F. Bunshi, A. Yokoyama, K. Tamura, T. Asakura, M. Uo, K. Motomiya, B. Jeyadevan, R. Hatakeyama, W. Fumio and K. Tohji, Mol. BioSyst. 1, 142 (2005).

[17] A. J. Stone and D. J. Wales, Chem. Phys. Lett. 128, 501 (1986).

[18] L. G. Zhou and S. Q. Shi, Carbon 41, 579 (2003).

[19] Gaussian 03, Revision C.02, M. J. Frisch, G. W. Trucks, H. B. Schlegel, G. E. Scuseria, M. A. Robb, J. R. Cheeseman, J. A. Montgomery, Jr., T. Vreven, K. N. Kudin, J. C. Burant, J. M. Millam, S. S. Iyengar, J. Tomasi, V. Barone, B. Mennucci, M. Cossi, G. Scalmani, N. Rega, G. A. Petersson, H. Nakatsuji, M. Hada, M. Ehara, K. Toyota, R. Fukuda, J. Hasegawa, M. Ishida, T. Nakajima, Y. Honda, O. Kitao, H. Nakai, M. Klene, X. Li, J. E. Knox, H. P. Hratchian, J. B. Cross, V. Bakken, C. Adamo, J.
Jaramillo, R. Gomperts, R. E. Stratmann, O. Yazyev, A. J. Austin, R. Cammi, C. Pomelli, J. W. Ochterski, P. Y. Ayala, K. Morokuma, G. A. Voth, P. Salvador, J. J. Dannenberg, V. G. Zakrzewski, S. Dapprich, A. D. Daniels, M. C. Strain, O. Farkas, D. K. Malick, A. D. Rabuck, K. Raghavachari, J. B. Foresman, J. V. Ortiz, Q. Cui, A. G. Baboul, S. Clifford, J. Cioslowski, B. B. Stefanov, G. Liu, A. Liashenko, P. Piskorz, I. Komaromi, R. L. Martin, D. J. Fox, T. Keith, M. A. Al-Laham, C. Y. Peng, A. Nanayakkara, M. Challacombe, P. M. W. Gill, B. Johnson, W. Chen, M. W. Wong, C. Gonzalez, and J. A. Pople, (Gaussian, Inc., Wallingford CT, 2004).

[20] D. Braga, F. Grepioni and A. G. Orpen, Organometallics 12, 1481 (1993).

[21] NBO Version 3.1, E. D. Glendening, A. E. Reed, J. E. Carpenter and F. Weinhold. 